\title{
Stochastic Analysis of Multi-tier Nanonetworks in THz Band
}

\author{
Joonas Kokkoniemi, Janne Lehtomäki, and Markku Juntti \\ Centre for Wireless Communications, University of Oulu \\ P.O. Box 4500, 90014 Oulu, Finland \\ forename.surname@oulu.fi
}

\begin{abstract}
Future nanonetworks are formed by large numbers of autonomous, nano-sized sensors. These are often envisioned to be paired with one or more layers of higher complexity devices, providing access to the external networks. The number of devices sharing the same frequency resources can theoretically be very high, up to several hundreds per square meter. This causes the overall interference of the network to increase with the complexity of the network. In this work, stochastic geometry is utilized to derive the moments of the summed interference in the case of multi-tier nanonetworks in the terahertz frequency band $(0.1-10 \mathrm{THz})$. All the devices in all the tiers of the network are assumed to be Poisson distributed. Based on this assumption, models for the moments of interference are derived and they are shown by computer simulations to predict the mean interference and its higher moments exactly.
\end{abstract}

\section{CCS CONCEPTS}

-Networks $\rightarrow$ Network performance modeling; Network simulations;

\section{KEYWORDS}

Mean interference, stochastic geometry, THz band

\section{ACM Reference format:}

Joonas Kokkoniemi, Janne Lehtomäki, and Markku Juntti. 2 017. Stochastic Analysis of Multi-tier Nanonetworks in THz Band. In Proceedings of NANOCOM '17, Washington D.C., DC, USA, September 27-29, 2017, 7 pages.

\section{INTRODUCTION}

Wireless sensor networks are very important part of the future communication systems [5]. The main reason for this is the ever and exponentially increasing number of Internet-of-Things (IoT) devices. Interconnected to the Internet, these devices, and networks of them, can provide a vast amount of information of our surrounding environment. This information can be used in variety of applications, such as road safety, surveillance, remote controlling, and buildings' climate control, just to mention few. The number of possible applications for the IoT devices is endless and mostly up to imagination.
Advances in the material sciences have brought nanodevices and nanonetworks a step closer to reality [1]. These very small devices have mostly applications in sensing tasks, but also in drug delivery and actuation [1]. They can be utilized in similar applications as their larger counterparts, but small size makes it possible to attach sensors and also networking capabilities to practically to any object. There is still plenty of research work to be done before we see actual nanodevices in the markets, but the large amount of resources put to the research on nano-sized electronics components ensures they will be reality in the future.

One very popular frequency band for a communication medium for these very small devices is the terahertz band (0.1-10 THz). It has been considered because of the miniature antennas would force the center frequencies of the transmissions very high, but on the other hand, with novel graphene-based solutions the $\mathrm{THz}$ band will become a feasible medium for wireless communications [10]. Second reason for popularity of the $\mathrm{THz}$ band are the potential extremely high data rate macroscopic applications for this vast unlicensed band. Most of the research on channel behavior is valid for any scale of communications, such as the stochastic modeling of the interference in this paper. The downside of the THz band is a large path loss and molecular absorption, which limit the maximum communication distances [9]

This paper addresses the interference characterization of dense $\mathrm{THz}$ band networks using stochastic geometry. This is an extension to our previous paper on general stochastic modeling of the interference in simple Poisson distributed networks [12]. In this paper, we assume multi-tier network and extend the expressions for the moments of interference. By multi-tier, we mean that the network is formed of multiple parallel node categories, such as base stations (BSs) and sensor nodes (SNs), sharing the same spectral resources. By extending the models in [12], we provide an easy way to estimate network performance given the tier, and transmitter (Tx) and receiver ( $\mathrm{Rx})$ characteristics. The main focus here is on nanonetworks, but the interference models are derived in a general manner, such that the analysis is feasible for any network following our assumptions.

Stochastic geometry is a powerful tool for studying network level interference as well as stochastic processes in general. There are numerous papers written about using stochastic geometry in estimating the interference in wireless networks, such as $[2-4,6-$ 8]. Our model for the $\mathrm{THz}$ band is relying on similar approach to those, but because the channel model is different to most papers, we confirm all the models with computer simulations. Still, the ultimate goal of the stochastic geometry is to evaluate the network performance without a need for large network simulations that take time and resources.

There are few works on stochastic geometry specifically for the $\mathrm{THz}$ frequencies [13-15]. Those works have two major difference 
to this paper, as well as to our previous paper on the stochastic geometry for THz band networks [12]. Firstly, those assume Matern's hard-core process, which assumes certain guard bands around the Txs and Rxs. This is one form of a dependent thinning of a Poisson distributed network and is very useful in real-life network deployment scenarios where distances between the nodes can be controlled. For instance, a $\mathrm{THz}$ access point network and users inside a room. We, on the other hand, assume fully random network deployment, e.g., a large group of nanodevices randomly thrown in environment. In this situation a regular Poisson process better describes the network deployment.

Second major difference c omes from the u tilization of a selfinduced noise in the model. This noise component has been predicted to exist as a consequence of reradiation of the absorbed energy. However, the commonly utilized model for it has some major issues, which changes the received signal and interference behavior drastically. These issues will be detailed in the next section.

\section{SYSTEM MODEL}

We consider $K$-tier network, i.e., the total network of nodes consists $K$ independently distributed sets of nodes. These tiers may represent, e.g., different d evice $\mathrm{c}$ ategories, such a s b ase stations and sensor nodes, as we utilize them in numerical results. The layered structure of the network is a handy assumption if we have different node densities, transmit powers, antenna characteristics, etc. in different device categories sharing the same spectral resources. All the nodes are assumed to follow Poisson distribution. Therefore, each tier $k$ consist in average $N_{k}$ nodes drawn from Poisson distribution, and thus, each tier has it characteristic node density

$\lambda_{k}$, for which $N_{k}=\lambda_{k}|B|$, where $|B|$ is certain area/volume for which the $N_{k}$ nodes have been distributed on.

We assume circularly/spherically symmetric space around the origin, where a typical node resides. This node therefore sees the average network behavior as the nodes of the network are distributed around it. More information of the assumptions and properties of this type of a network can be found, e.g., in our previous paper about the stochastic geometry for the $\mathrm{THz}$ band [12].

Fig. 1 gives an illustration of a two layer system, similar to one for which we give the numerical results for. In this system, we consider two device categories: sensor nodes (SNs) and base stations (BSs). SNs are simple isotropic broadcasters $\left(\mathrm{Tx}_{\mathrm{S}}\right)$. BSs $\left(\mathrm{Tx}_{\mathrm{b}}\right.$ and $\mathrm{Rx}_{\mathrm{b}}$ ) are utilizing directional antennas in transmission, but receive isotropically. Because all the nodes are assumed to receive isotropically, also the typical node at the origin is isotropic. This illustrates a scenario where BSs are pairwise connected at a given time and the SNs are blindly broadcasting their sensing information. All the nodes are randomly distributed, as are the antenna directions in the case of BSs. As the theoretical modeling considers interference, only the transmitters are modeled in the mathematical model (receiving does not induce interference to the network).

\subsection{Path Loss Model}

We assume very common path loss model used in $\mathrm{THz}$ communication specific papers. It consists free space path loss and molecu-

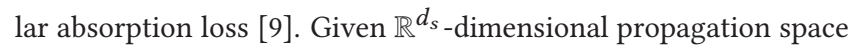

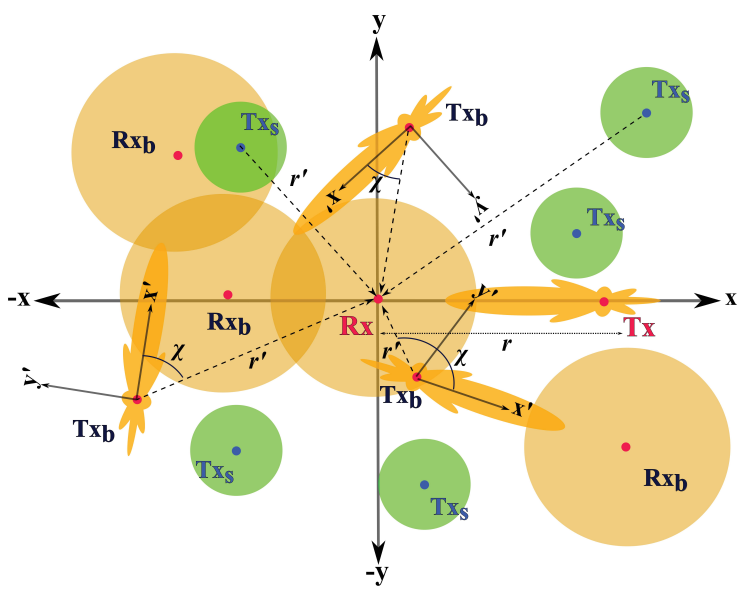

Figure 1: The system model with a typical node at the origin and the corresponding desired transmitter on the horizontal axis. The base stations utilize directional beams in transmissions. The other nanosensor utilize isotropic antennas in their transmissions.

[17], the path loss model is given by

$$
l(r)=\frac{\exp \left(-\kappa_{\mathrm{a}}(f) r\right)}{c_{d_{s}} d_{s} r^{d_{s}-1}} A,
$$

where $\kappa_{\mathrm{a}}(f)$ is the absorption coefficient, or a sum of loss coefficients in the case multiple loss mechanisms, $A$ is the antenna aperture term in $d_{s}$-dimensional space, $r$ is the distance from Tx to $\mathrm{Rx}$, and $c_{d_{s}}$ is the volume of $d_{s}$-dimensional unit ball [17]:

$$
c_{d_{s}}= \begin{cases}\frac{\pi^{\frac{d_{s}}{2}}}{\left(d_{s} / 2\right) !} & d_{s} \text { even } \\ \frac{1}{d_{s} !} \pi^{\frac{d_{s}-1}{2}} 2^{d_{s}}\left(\frac{d_{s}-1}{2}\right) !, & d_{s} \text { odd } .\end{cases}
$$

In any practical applications $c_{d_{s}}=\pi$ in $\mathbb{R}^{2}$ and $c_{d_{s}}=\frac{4}{3} \pi$ in $\mathbb{R}^{3}$. The absorption coefficient can be calculated with databases, such as the HITRAN database [16].

\subsection{Interference and Noise}

Utilizing the above path loss model, the aggregate interference of the layer $k$ becomes

$$
I_{\mathrm{aggr}, \mathrm{k}}(f)=\sum_{i \in \Phi_{k}} P_{\mathrm{Tx}, \mathrm{k}} \mathbb{E}_{\Theta}\left[G_{\mathrm{Tx}, \mathrm{k}}(\Theta)\right] \mathbb{E}_{\Theta}\left[G_{\mathrm{Rx}, \mathrm{k}}(\Theta)\right] l\left(r_{i}\right),
$$

where $\Phi_{k}$ is the set of interfering nodes, $P_{\mathrm{Tx}, \mathrm{k}}$ is the transmit power of tier $k$ Txs, $\mathbb{E}_{\Theta}\left[G_{\mathrm{Tx}, \mathrm{k}}(\Theta)\right]$ and $\mathbb{E}_{\Theta}\left[G_{\mathrm{Rx}, \mathrm{k}}(\Theta)\right]$ are the expected antenna gains of the Txs and the typical $\mathrm{Rx}$, and $\Theta$ is the orientation

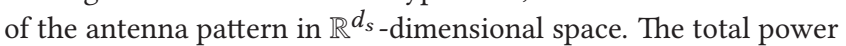
function of a single device is henceforth marked as

$$
l_{k}\left(r_{i}\right)=P_{\mathrm{Tx}, \mathrm{k}} \mathbb{E}_{\Theta}\left[G_{\mathrm{Tx}, \mathrm{k}}(\Theta)\right] \mathbb{E}_{\Theta}\left[G_{\mathrm{Rx}, \mathrm{k}}(\Theta)\right] l\left(r_{i}\right) .
$$

Given the above aggregate interference and path loss for the desired signal, signal-to-interference-plus-noise ratio (SINR) becomes

$$
\operatorname{SINR}(f)=\frac{S(f)}{N(f)+I_{\mathrm{aggr}, \mathrm{k}}(f)} \approx \frac{S(f)}{I_{\mathrm{aggr}, \mathrm{k}}(f)},
$$

where $S(f)=P_{\mathrm{Tx}} l(r)$ is the received desired signal and $N(f)$ is the noise power. The last term is signal-to-interference ratio (SIR) and 
follows from our assumption of the interference limited network, i.e., the noise has minimal contribution to the total interferenceplus-noise level.

There are numerous papers talking about self-induced noise, i.e., the noise caused by reradiation of the absorbed energy, which could theoretically cause contribution even in the interference limited networks. We have written a p aper a bout the b ehavior of the self-induced noise, where we also also derived the existing model for it [11]. This is given in most papers as $S_{N}(f)=P_{\mathrm{Tx}} l(r)(1-$ $\left.\exp \left(-\kappa_{\mathrm{a}} r\right)\right)$, where $\left(1-\exp \left(-\kappa_{\mathrm{a}} r\right)\right)$ is the emissivity of the medium. As we discussed already discussed in [11], besides the emissivity of the medium this model is completely wrong.

It is particularly bad assumption in stochastic models, since it erases the molecular absorption from the interference. If we write the the received power in terms of the above noise model and the desired signal, we obtain

$$
I_{R x}=I_{T x}(1-\exp (-\kappa r)) l(r)+I_{T x} \exp (-\kappa r) l(r) \equiv I_{T x} l(r),
$$

and thus, there is no molecular absorption. This also occurs for the summed interference, and thus, it changes the interference distribution! Furthermore, assuming a large transmit power, i.e., we can safely disregard regular noise from single link's signal-to-noise ratio (SNR). Calculating this reveals that

$$
\begin{aligned}
\mathrm{SNR} & =P_{\mathrm{Tx}} l(r) / P_{\mathrm{Tx}} l(r)\left(1-\exp \left(-\kappa_{\mathrm{a}} r\right)\right) \\
& \equiv \exp \left(-\kappa_{\mathrm{a}}(f) r\right) /\left(1-\exp \left(-\kappa_{\mathrm{a}} r\right)\right),
\end{aligned}
$$

which simply states that SNR of a single link does not experience any spreading loss, even in the case of isotropic antennas and regardless of the distance between the transmitter and receiver, nor does the SNR depend on the transmit power!

The reason for this misleading behavior lies in the source term of this noise model. The source of the noise is not at the origin, but in the surrounding medium of the receiver. Thus, using this noise model only leads to misleading results as detailed above and should be avoided at all cost. As we concluded in [11], the true contribution of the self-induced noise is most likely very small. As a consequence, throughout this paper we assume that the network is interference limited and give metrics for the interference only. However, it should be noticed that if the network is thin enough, interference limited network assumption no longer holds and appropriate noise terms should be added in the equations.

\subsection{Directional Antennas}

Antenna effects can be taken into account by calculating the expected antenna gain over the antenna radiation pattern in $\mathbb{R}^{d_{s}}$ dimensional space. Here we assume that the antenna patterns $(\mathrm{Rx}$ and Tx) $G(\Theta)$ always integrate to unity. In the other words, we assume that the radiated unit transmit power remains constant regardless of the antenna pattern, i.e., the antenna does not increase or decrease the total radiated power. Thus, the expected antenna gain becomes

$$
\mathbb{E}_{\Theta}[G(\Theta)]=\frac{1}{c_{d_{s}} d_{s}} \int_{\mathbb{R}^{d_{s}}} G(\Theta) d \mathbb{R}^{d_{s}}=\frac{1}{c_{d_{s}} d_{s}},
$$

where $1 / c_{d_{s}} d_{s}$ is the effective expected antenna gain, because the radiated unit transmit power is constant and the total gain is assumed to integrate to unity. From this expression it can be seen that if the radiated unit transmit power is kept constant, the antenna pattern has no impact on the mean interference power. However, the antenna pattern has an impact on the higher moments of interference. This will be detailed in the next section.

As mentioned above, in the numerical results we assume two layer network where SNs utilize isotropic antennas and BSs use directional antennas. For directional antennas we use simple cone shaped antenna pattern that gives strong gain forward without any side lobes. This is mainly because of the mathematical simplicity of this pattern, but nothing in the theories forbid of using more complex antenna patterns. Complex antenna patterns mainly make the simulations more difficult, since as it was stated above, antenna patterns do not have any effect on the mean interference and the higher moments can be handled rather easily as it will shown in the next section.

\section{STOCHASTIC MODELS}

Considering $K$-tier network and the path loss models given above, the aggregate interference of the network is given by

$$
I_{\text {aggr }}(f)=\sum_{k=1}^{K} \sum_{i \in \Phi_{k}} l_{k}\left(r_{i}\right)
$$

where the sum over $K$ tiers is done based on the independence of the tiers, i.e., superimposing multiple Poisson point processes (PPPs) is still a PPP [2, Sec. 1.3]. Using this expression for the aggregate interference, we can calculate the moments by first calculating the Laplace transform of (9). It is given by $[7,8]$

$$
\mathcal{L}_{I_{\text {aggr }}}(s)=\mathbb{E}_{\Phi}\left[\exp \left(-s \sum_{k=1}^{K} \sum_{i \in \Phi_{k}} l_{k}\left(r_{i}\right)\right)\right],
$$

where $\Phi$ indicates expectation over all sets of nodes $\Phi_{k}$. Because of the independence of tiers, above equation can also be given as

$$
\mathcal{L}_{I_{\text {aggr }}}(s)=\prod_{K} \mathbb{E}_{\Phi_{k}}\left[\prod_{i \in \Phi_{k}} \exp \left(-s l_{k}\left(r_{i}\right)\right)\right] .
$$

where term

$$
\Omega(v)=\mathbb{E}_{\Phi_{k}}\left[\prod_{i \in \Phi_{k}} \exp \left(-s l_{k}\left(r_{i}\right)\right)\right]=\mathbb{E}_{\Phi_{k}}\left[\prod_{x \in \Phi_{k}} v(x)\right]
$$

is called the probability generating functional (PGFL) [8], where $v(x)=\exp \left(-s l_{k}\left(r_{x}\right)\right)$. We can solve this expectation for PPP as [8]

$$
\Omega(v)=\exp \left(-\int_{\mathbb{R}^{d}}(1-v(x)) \Lambda_{k}(x) d x\right),
$$

where $d$ is the dimension of space and $\Lambda_{k}(x)$ is the intensity function of the PPP of tier $k$. If the nodes in the network are homogenously distributed, i.e., the Poisson process is homogenous,

$$
\Lambda_{k}(x)=p_{k} c_{d_{d}} d_{d} \lambda_{k} x^{d_{d}-1},
$$

with a fixed intensity parameter $\lambda_{k}$ over all distances, where $p_{k}$ is the probability of a node to transmit (an effective thinning operation for tier $k$ ), $c_{d_{d}}$ and $d_{d}$ are a unit volume and dimension of the node drop space. By separating the propagation and the node drop spaces, we can derive far more general model, where nodes 
can be dropped on a plane or volume, whereas propagation space (or the path loss exponent only) can be of higher or lower dimension. Thus, $c d_{s}$ and $d_{s}$ explicitly quantify the unit volume and the dimension of the propagation space. Furthermore, it would also be possible to use independent node drop dimensions and propagation space dimensions for different tiers $k$, but for simplicity, all the nodes are assumed to share the same space.

Getting back to the Laplace transform, due to usage of the unit area term $c_{d_{d}} d_{d}$ and an assumption of circularly/sphrically symmetric space, the integral over $\mathbb{R}^{d_{d}}$ is just an integration over the distance $r$. The Laplace transform becomes

$$
\mathcal{L}_{I_{\mathrm{aggr}}}(s)=\prod_{K} \exp \left[-p_{k} c_{d_{d}} d_{d} \lambda_{k} \int_{0}^{\infty}\left(1-\exp \left(-s l_{k}(r)\right)\right) r^{d_{d}-1} d r\right] \text {. }
$$

Now we have an expression from which we can calculate the moments of the interference. The $n$th raw moment of the aggregate interference power can be calculated from the $n$th derivative of the Laplace transform by [4]

$$
\mathbb{E}\left[I^{n}\right]=\left.(-1)^{n} \frac{d^{n}}{d s^{n}} \mathcal{L}_{I_{\text {aggr }}}(s)\right|_{s=0} .
$$

We can simplify the expressions for the moments by marking Laplace transform in (15) as

$$
\mathcal{L}_{I_{\text {aggr }}}(s)=\exp (-L(s))
$$

where

$$
L(s)=\sum_{K} p_{k} c_{d_{d}} d_{d} \lambda_{k} \int_{0}^{\infty}\left(1-\exp \left(-s l_{k}(r)\right)\right) r^{d_{d}-1} d r .
$$

By calculating the derivatives in (16), the first three moments become:

$$
\begin{gathered}
\mathbb{E}\left[I_{\text {aggr }}\right]=L^{\prime}, \\
\mathbb{E}\left[I_{\text {aggr }}^{2}\right]=-L^{\prime \prime}+\left(L^{\prime}\right)^{2}, \\
\mathbb{E}\left[I_{\text {aggr }}^{3}\right]=L^{\prime \prime \prime}-3 L^{\prime \prime} L^{\prime}+\left(L^{\prime}\right)^{3},
\end{gathered}
$$

where

$$
\begin{aligned}
L^{\prime}(s=0) & =\sum_{K} p_{k} \lambda_{k} A P_{\mathrm{Tx}, \mathrm{k}}(f) \mathbb{E}_{\Theta}\left[G_{\mathrm{Tx}, \mathrm{k}}(\Theta)\right] \mathbb{E}_{\Theta}\left[G_{\mathrm{Rx}, \mathrm{k}}(\Theta)\right] \\
& \times \frac{c_{d_{d}} d_{d}}{c_{d_{s}} d_{s}} \int_{0}^{\infty} r^{d_{d}-d_{s}} \exp \left(-\kappa_{\mathrm{a}}(f) r\right) d r \\
L^{\prime \prime}(s=0) & =-\sum_{K} p_{k} \lambda_{k}\left(A P_{\mathrm{Tx}, \mathrm{k}}(f) \mathbb{E}_{\Theta}\left[G_{\mathrm{Tx}, \mathrm{k}}(\Theta)\right] \mathbb{E}_{\Theta}\left[G_{\mathrm{Rx}, \mathrm{k}}(\Theta)\right]\right)^{2} \\
& \times \frac{c_{d_{d}} d_{d}}{\left(c_{d_{s}} d_{s}\right)^{2}} \int_{0}^{\infty} r^{d_{d}-2 d_{s}+1} \exp \left(-2 \kappa_{\mathrm{a}}(f) r\right) d r
\end{aligned}
$$

and

$$
\begin{aligned}
L^{\prime \prime \prime}(s=0) & =\sum_{K} p_{k} \lambda_{k}\left(A P_{\mathrm{Tx}, \mathrm{k}}(f) \mathbb{E}_{\Theta}\left[G_{\mathrm{Tx}, \mathrm{k}}(\Theta)\right] \mathbb{E}_{\Theta}\left[G_{\mathrm{Rx}, \mathrm{k}}(\Theta)\right]\right)^{3} \\
& \times \frac{c_{d_{d}} d_{d}}{\left(c_{d_{s}} d_{s}\right)^{3}} \int_{0}^{\infty} r^{d_{d}-3 d_{s}+2} \exp \left(-3 \kappa_{\mathrm{a}}(f) r\right) d r .
\end{aligned}
$$

Any higher moments can be obtained in similarly.

The mean aggregate interference can be solved in the case of $d_{s} \leq d_{d}$ as

$$
\begin{aligned}
\mathbb{E}\left[I_{\mathrm{aggr}}\right] & =\sum_{K} P_{\mathrm{Tx}, \mathrm{k}}(f) \mathbb{E}_{\Theta}\left[G_{\mathrm{Tx}, \mathrm{k}}(\Theta)\right] \mathbb{E}_{\Theta}\left[G_{\mathrm{Rx}, \mathrm{k}}(\Theta)\right] \\
& \times p_{k} \lambda_{k} \frac{c_{d_{d}} d_{d}}{c_{d_{s}} d_{s}} \frac{\left(d_{d}-d_{s}\right) !}{\kappa_{\mathrm{a}}^{d_{d}-d_{s}+1}(f)}
\end{aligned}
$$

where $\left(d_{d}-d_{s}\right)$ ! is replaced with $\Gamma\left(d_{d}-d_{s}+1\right)$, where $\Gamma(\cdot)$ is the gamma function, if path loss exponent has non-integer value. If $d_{s}>d_{d}$, the mean interference level cannot be solved, but requires numerical approximation. Moreover, if $d_{s}>d_{d}$, the path loss has a singularity around the typical node and requires lower bounding the integral in (22). If $d_{s} \leq d_{d}$, there are no singularities as it can be seen in the above expressions. However, in this case, if the absorption coefficient approaches zero, solution in (25) cannot be used, since the interference approaches infinity. In the case of small absorption coefficient with $d_{s} \leq d_{d}$, (22) should be utilized. This is because without the molecular absorption, interference does not die fast enough with distance and the only finite solutions are given by finite area approximations by upper bounding the integral.

We can also solve variance of the aggregate interference by noticing that the variance in the terms of raw moments is simply

$$
\operatorname{var}\left(I_{\text {aggr }}\right)=-L^{\prime \prime}(s=0),
$$

because $\operatorname{var}(X)=\mathbb{E}\left[X^{2}\right]-(\mathbb{E}[X])^{2}$, and thus, yields the above expression when we subtract the square of (19) from (20). Higher central moments have more difficult expressions, but can be solved similarly based on the raw moments.

As it was mentioned in the previous section, antenna patterns have no impact on the mean interference if the total radiated power of an antenna pattern is fixed. However, they do have an impact on the higher moments because the maximum gain given by antenna linearly scales the transmit power. Therefore, the maximum effective transmit power varies between zero and

$$
P_{\mathrm{Tx}, \mathrm{k}}(f) \max _{\Theta}\left[G_{\mathrm{Tx}, \mathrm{k}}(\Theta)\right] \max _{\Theta}\left[G_{\mathrm{Rx}, \mathrm{k}}(\Theta)\right] .
$$

As a consequence, the derivatives of Laplace transform need to be multiplied with a gain factor

$$
f_{G}=\left(\frac{\max _{\Theta}\left[G_{\mathrm{Tx}, \mathrm{k}}(\Theta)\right] \max _{\Theta}\left[G_{\mathrm{Rx}, \mathrm{k}}(\Theta)\right]}{\mathbb{E}_{\Theta}\left[G_{\mathrm{Tx}, \mathrm{k}}(\Theta)\right] \mathbb{E}_{\Theta}\left[G_{\mathrm{Rx}, \mathrm{k}}(\Theta)\right]}\right)^{n-1},
$$

where $n$ is the derivative order. This follows from the fact that starting from the second moment, the variance of the received power has an impact on the moments and the transmit power increases in its $n$th power we proceed to higher moments. Therefore, increased variability is also affected by the maximum antenna gains. We can see that in the case of isotropic antennas, the above term is always one. Furthermore, we can see in the numerical results that this expression predicts the higher moments exactly as we utilize directional antennas for the BSs in simulations and take the above equation into account in the theoretical calculations.

\section{NUMERICAL RESULTS}

Some numerical examples for the moments of the aggregate interference are given in this section. They are compared to computer 


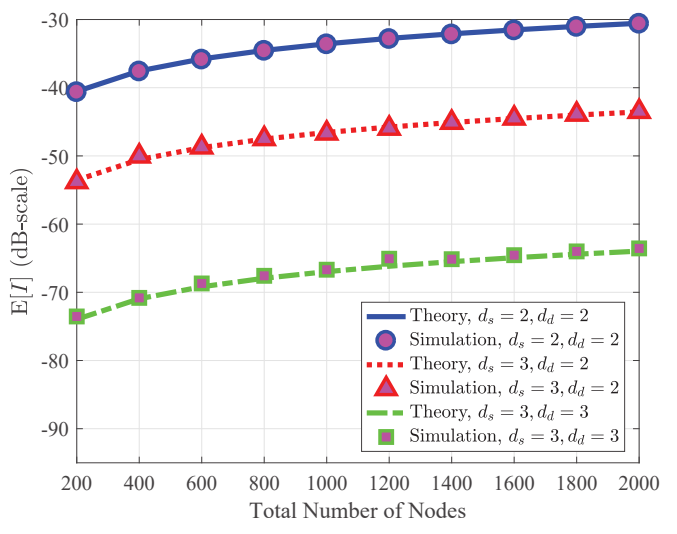

Figure 2: Aggregate interference with various node drop and path loss dimensions.

simulations in order to confirm the validity of the derived models. The simulation parameters for the BSs and SNs were chose in way that they combine different characteristics of the theories. They were chose as: transmit power $P_{\mathrm{Tx}}=[1,1]([\mathrm{BSs}, \mathrm{SNs}])$, probability to transmit $p=[0.5,1]$, and number of nodes $N=$ $[50-50,150-1500]$. As it was mentioned above, BSs use directional antennas and NSs are isotropic. Furthermore, absorption coefficient $\kappa_{\mathrm{a}}$ was $0.1 \mathrm{~cm}^{-1}$, unless the interference was simulated over frequencies. This absorption coefficient is roughly the same as is the peak absorption at approximately $1.1 \mathrm{THz}$. Simulation area had a radius of $400 \mathrm{~cm}$, which makes sure there is no contribution to the interference outside this area with sufficient absorbtion. All the simulations were done over one million network realizations, expect for the three dimensional path loss and three dimensional node drop case, which was simulated over four million realizations, and the frequency domain results, which have been obtained over 50,000 network realizations and over the whole THz band. Directional antennas had a $\pi / 4$ wide cone-shaped antenna pattern (see figure in [12]).

\subsection{Simulation Setup}

The simulator in this paper was a simple distance-based Matlabbased Monte Carlo simulator. That is, the distances and the antenna orientations were randomly distributed, and were fed to the channel model. Antenna patterns require special attention as the dimension of the propagation and drop space, as well as the pattern itself have impact on the results. These are went through in detail in [12] for very similar Monte Carlo simulation case. However, one should notice different assumptions on antenna directivities, i.e., here we consider BSs to send with directional antennas which are received with isotropic antennas, but otherwise the simulation model for a single layer is the same in both papers.

\subsection{The Moments of Interference}

Figs. 2 to 4 show the first three moments of the interference as a function the number of users for the most interesting dimension combinations. We can see that the curves match perfectly up until the second and third moment of three dimensional path loss with three dimensional node drop, although, those were simulated with

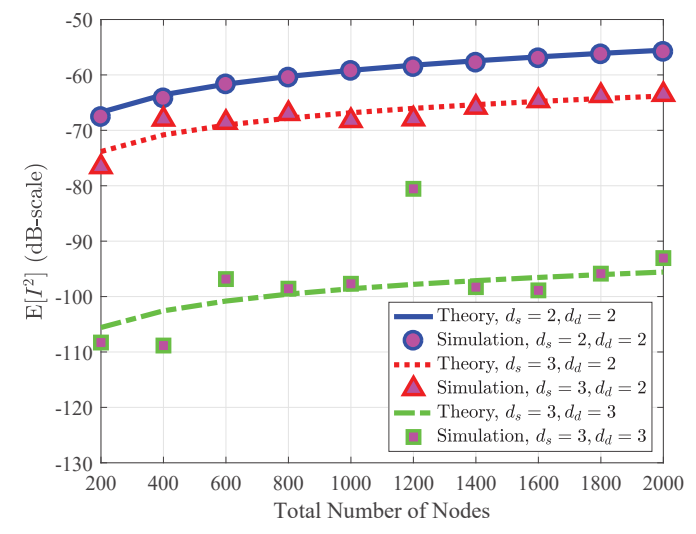

Figure 3: Second raw moment of the aggregate interference with various node drop and path loss dimensions.

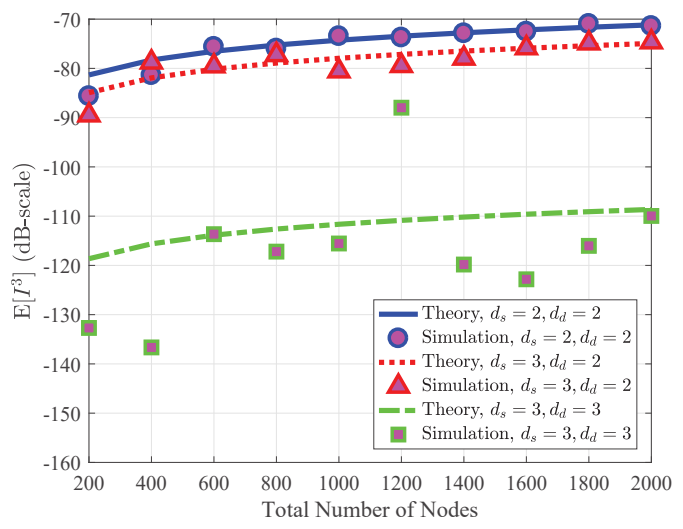

Figure 4: Third raw moment of the aggregate interference with various node drop and path loss dimensions.

larger number of realizations. This is purely simulation specific issue since we keep the number of users fixed over all dimensions. This causes the network density to be lowest for the three dimensional node drop. But even more importantly, as we progress towards higher moments, the more variance there is in the results. Pushing the simulation model any further would cause the simulations to last several days, or weeks. This is a perfect example of the power of the stochastic geometry: the same estimation with more accuracy is obtained instantaneously! This truly advertises the utilization of the stochastic geometry over complicated simulation models, which on their behalf, aim at the stochastic average values that can be obtained very easily as it has been shown, e.g., in this paper. As it was mentioned earlier, the directional antennas have an impact on the variance of interference, and therefore, on the higher moments. By introducing the gain factor (27), the theoretical results give a perfect match to the simulated ones.

\subsection{Frequency Domain SIR}

The frequency domain results in Figs. 5 and 6 give SIR values. We earlier mentioned about the problem of the mean interference approaching infinity as the absorption coefficient approaches zero. This occurs on various frequencies where absorption is very low, 


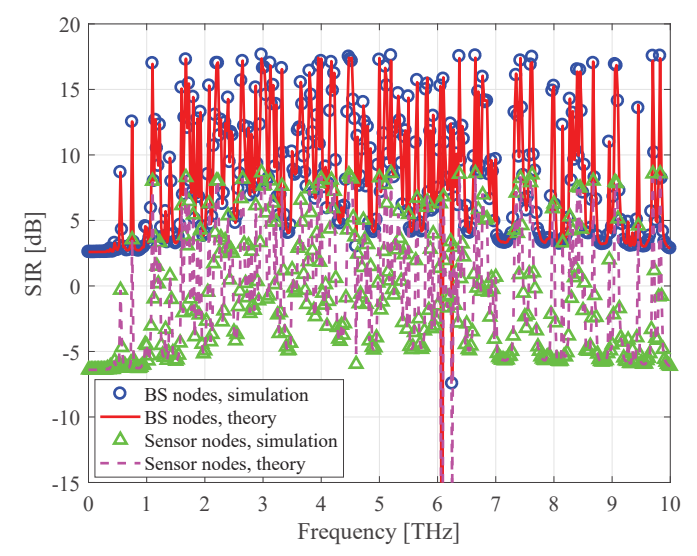

Figure 5: SIR performance as a function of frequency with 50 BSs and 150 SNs in 2D path loss and 2D node drop case.

e.g., at the lower frequencies below $300 \mathrm{GHz}$. Therefore, the theoretical results are calculated with (22) even if the exact solution would have been available. This way the upper boundary of the distance in theory was limited to the same $400 \mathrm{~cm}$ as was the radius of the simulation area. By doing this, results match perfectly as they should based on the theories.

SIR values were calculated separately for BSs and SNs at $5 \mathrm{~cm}$ distance using the total aggregate interference for interfering links and the path loss in (1) with appropriate antenna gains for the desired links. The desired transmitting BS was assumed to use directional antenna, whereas the desired SN was using isotropic antenna. As expected, the link with directional antenna experiences far lower loss and the service would be much easier to guarantee. However, we should remember that the results here are only some examples of what the theoretical models can do. Therefore, making further conclusions of the results, outside the fact that theoretical models match perfectly with the simulation results, is useless. Still, the frequency domain figures show that the harmful molecular absorption also acts as a shield against the interference and may provide some benefit in dense $\mathrm{THz}$ band networks.

\section{CONCLUSION}

We derived expressions for the moments of interference in $\mathrm{THz}$ networks for the commonly utilized channel model in $\mathrm{THz}$ band communications. These expressions can be utilized in arbitrarydimensional space and arbitrary-dimensional path loss, and they can be easily extended to calculate any possible moment of the aggregate interference. The theoretical models were compared against computer simulations that showed perfect fit within the simulation accuracy. The moments of the interference have wide range of use cases among interference characterization in nanonetworks, as well as in macroscopic networks. They are essential when estimating the interference distributions and general network performance. Utilizing stochastic models instead of simulations save time and provide equal or better accuracy. After all, in the end, simulation models aim to achieve result of the stochastic models. Thus, by utilizing mathematical models, one can skip the simulations all together and jump straight to the solutions.

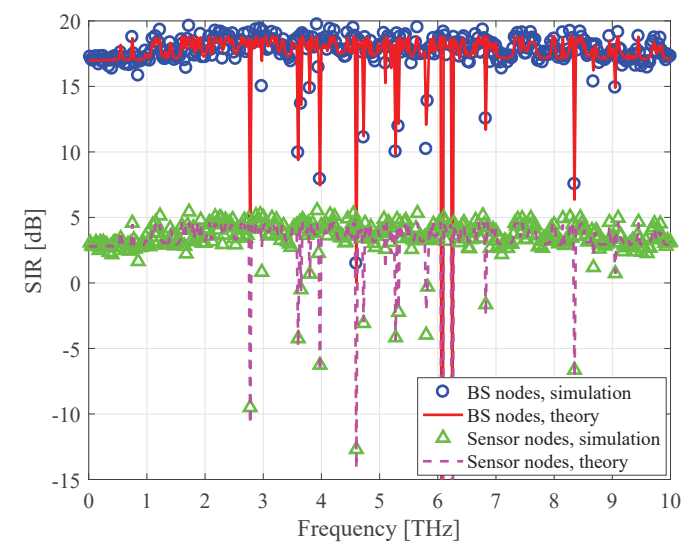

Figure 6: SIR performance as a function of frequency with 50 BSs and 150 SNs in 3D path loss and 2D node drop case.

\section{REFERENCES}

[1] I. F. Akyildiz and J. M. Jornet. Electromagnetic wireless nanosensor networks. Elsevier Nano Commun. Networks 7., 1(1):3-19, Mar. 2010.

[2] F. Baccelli and B. Blaszczyszyn. Stochastic geometry and wireless networks, volume I - Theory. Foundations and Trends in Networking, 3(3-4):249-449, Dec. 2009.

[3] A. Baddeley. Stochastic Geometry: Spatial Point Processes and their Applications. Lecture Notes in Mathematics. Springer Berlin Heidelberg, 2007.

[4] H. ElSawy, E. Hossain, and M. Haenggi. Stochastic geometry for modeling, analysis, and design of multi-tier and cognitive cellular wireless networks: A survey. IEEE Commun. Surveys Tuts., 15(3):996-1019, Jun. 2013.

[5] G. P. Fettweis. 5G and the future of IoT. In Proc. European Solid-State Circuits Conf., pages 21-24, 2016.

[6] M. Haenggi. Outage, local throughput, and capacity of random wireless networks. IEEE Trans. Wireless Commun., 8(8):4350-4359, Aug. 2009.

[7] M. Haenggi, J. G. Andrews, F. Baccelli, O. Dousse, and M. Franceschetti. Stochastic geometry and random graphs for the analysis and design of wireless networks. IEEE 7. Sel. Areas Commun., 27(7):1029-1046, Sep. 2009.

[8] M. Haenggi and R. K. Ganti. Interference in large wireless networks. Foundations and Trends in Networking, 3(2):127-248, Nov. 2008.

[9] J. M. Jornet and I. F. Akyildiz. Channel modeling and capacity analysis for electromagnetic nanonetworks in the terahertz band. IEEE Trans. Wireless Commun., 10(10):3211-3221, Oct. 2011.

[10] J. M. Jornet and I. F. Akyildiz. Graphene-based plasmonic nano-antenna for terahertz band communication in nanonetworks. IEEE 7. Sel. Areas Commun., 31(12):685-694, Dec. 2013.

[11] J. Kokkoniemi, J. Lehtomäki, and M. Juntti. A discussion on molecular absorption noise in the terahertz band. Elsevier Nano Commun. Networks 7., 8:35-45, Jun. 2016.

[12] J. Kokkoniemi, J. Lehtomäki, and M. Juntti. Stochastic geometry analysis for mean interference power and outage probability in thz networks. [in press] IEEE Trans. Wireless Commun., pages 1-11, 2017.

[13] V. Petrov, M. Komarov, D. Moltchanov, J. M. Jornet, and Y. Koucheryavy. Interference and sinr in millimeter wave and terahertz communication systems with blocking and directional antennas. IEEE Trans. Wireless Commun., 16(3):17911808, Mar. 2017.

[14] V. Petrov, D. Moltchanov, and Y. Koucheryavy. Interference and SINR in dense terahertz networks. In Proc. IEEE Veh. Technol. Conf. (fall), pages 1-5, 2015.

[15] V. Petrov, D. Moltchanov, and Y. Koucheryavy. On the efficiency of spatial channel reuse in ultra-dense THz networks. In Proc. IEEE Global Commun. Conf., pages $1-7,2015$.

[16] L. S. Rothman et al. The HITRAN 2012 molecular spectroscopic database. F. Quant. Spectrosc. Radiat. Transfer, 130(1):4-50, Nov. 2013.

[17] S. Weber and J. G. Andrews. Transmission capacity of wireless networks. Foundations and Trends in Networking, 5(2-3):109-281, Jan. 2012. 